\title{
Recherche sur des larves de Nématodes Ascaridida trouvées chez Simulium damnosum
}

\author{
par O. BAIN et B. PHILIPPON
}

[Laboratoire de Zoologie (Vers) associé au C.N.R.S. Museum National d'Histoire Naturelle, (57, rue Cuvier, F 75 -Paris-5) et Centre Muraz - Section Onchocercose. Bobodioulasso (Haute-Volta)]

\begin{abstract}
Résumé
En Haute Volta, deux types de larves appartenant respectivement aux familles des Kathlanides et des Quimperides sont récoltées chez Simulium damnosum. Cet insecte ne joue pas sûrement le rôle d'un véritable hôte intermédiaire mais pourrait être un hôte d'attente.

Une nouvelle espèce de Quimperidae, Gendria leberrei n. sp., trouvée chez Bufo regularis est décrite bien qu'elle ne corresponde pas a priori aux larves de la même famille parasitant $S$. damnosum.
\end{abstract}

\section{Summary}

In Haute Volta two types of larvae belonging respectively to the families Kathlanides and Quimperides are collected in Simulium damnosum. This insect has not been proved to be true intermediate host; we believe it could be a resting host.

A new species of Quimperidae, Gendria leberrei n. sp. found in Bufo regularis is described although it does not belong \& a priori » to the family of the larvae parasiting $S$. damnosum. 
La présence de larves de Nématodes Secernentea, autres que des Filaires, chez Simulium damnosum Theobald 1903, a été signalée par divers auteurs (Lewis, 1953, Lewis et Marr, 1963, Marr et Lewis, 1964, Ovazza et coll., 1965) ; ces larves n'ont pu être identifiées, mais leur fréquence a fait l'objet d'études précises en vue d'étab'ir l'origine de certaines popu'ations simuliennes (Ba'ay, 1968).

Des recherches ont été effectuées au cours des mois de mai et juin 1968 en Haute Vo'ta, à la section Onchocercose du Centre Muraz, dans deux gites de savane guinéenne : l'un sur la Leraba (à Dagoidougou), l'autre sur la Volta Noire (à Samandeni). Dans cette région, les larves recueillies chez $S$. damnosum appartiennent à deux types. Il a paru utile d'en donner une description précise et de rechercher à quels adultes elles pouvaient correspondre.

\section{I. - Description des larves.}

\section{A. Larve de type Kathlanide (fig. $1 \mathrm{~A}$ à D).}

Ce type se rencontre chez les Simulies de la Leraba et de la Volta Noire. Bien que sa présence soit surtout connue chez les $q$, en raison du mode de capture sur appât humain des Simulies, il existe aussi chez les $\delta$ (un ơ éclos au laboratoire s'est révélé parasité, ce qui indique que les Simulies sont parasitées pendant leur vie larvaire aquatique) (1). Le taux d'infestation, calculé uniquement chez les femelles, est faible : 0,24 à $1,39 \%$ à la Leraba. En général, chaque Simulie renferme une seule larve, exceptionnellement deux ou trois.

Les larves sont libres dans les tubes de Malpighi et ne provoquent pas de réaction tissulaire ; elles sont très mobiles et pénètrent parfois lors des dissections dans l'estomac ou dans le proctodeum ; à l'anesthésie de la Simulie, elles peuvent être rejetées par l'anus.

Le corps de la larve est clair, mince, souvent enroulé en spirale et mesure en moyenne $700 \mu$ de long $(685 \mu$ chez le spécimen figuré) sur 22 à $25 \mu$ de large ; la région antérieure du corps s'amincit et se termine par trois lèvres saillantes exiguës (fig. $1 \mathrm{~A}$ ) ; la queue est effilée, incurvée ventralement, longue de $100 \mu$ et se termine de façon très caractéristique par une petite crosse dirigée vers la face postérieure (fig. $1 \mathrm{C}$ ). L'œsophage, long de $225 \mu$, débute par une portion étroite, régulièrement cylindrique, très allongée, présentant une différenciation pharyngée de $20 \mu$ de long et se termine par une portion renflée, dépourvue de granules de sécrétion et de valvules; cette portion terminale est relativement longue $(35 \mu)$, presque cylindrique, et présente parfois une légère constriction à mi-hauteur. La capsule buccale est réduite à un étroit tube cuticu'aire. Anneau nerveux et pore excréteur respectivement à $100 \mu$ et $160 \mu$ de

(1) Les 70 Simulies larvaires disséquées se sont révélées négatives, mais ceci s'explique aisément par la rareté du parasite. 
l'apex ; une grosse cellule visible sur la face ventrale du bulbe correspond probablement à la cellule excrétrice ; ébauche génitale à $210 \mu$ du début de l'intestin ; intestin à parois internes sinueuses et à brosse. Paroi du corps avec de nombreux et volumineux noyaux nettement visibles (fig. $1 \mathrm{~B}$ ).

Discussion. - Cette larve présente de grandes analogies avec celle d'un Kathlanide, Megalobatrachonema campanae Chabaud et Golvan 1957, recueillie chez un jeune Triton nouvellement infesté (2). La forme de la queue, la forme et la disposition des noyaux de la couche musculaire sont très proches ; l'œsophage présente les mêmes particularités : portion antérieure étroite, cylindrique, très allongée suivie d'un organite plus épais, sorte de bulbe sans valvule, particulièrement long; celui-ci pourrait résulter, comme chez les Kathlanides adultes, de la fusion du bulbe sensu stricto avec l'isthme élargi (la présence fréquente chez les spécimens d'une constriction à mi-hauteur de cet organite marquerait le passage du bulbe à l'isthme) (fig. $1 \mathrm{D}$ ).

\section{B. Larve de type Quimperide (fig. $1 \mathrm{E}$ à $\mathrm{H}$ ).}

Cette larve n'est actuellement connue qu'à la Leraba ; comme elle est très rare ( 2 positives sur environ 1.000 disséquées), son absence apparente à Samandeni peut être la conséquence d'un nombre insuffisant de dissections.

Sa localisation ne peut être précisée car elle a été recueillie uniquement en réjection proctodéale ; toutefois, on peut penser qu'eile reste dans la région postérieure du tube digestif et qu'elle peut même s'infiltrer dans les tubes de Malpighi.

Le corps de la larve est assez grand (1,85 mm de long) et épais (70 $\mu$ de large), avec deux ailes latérales se terminant un peu en avant des extrémités ; la tête porte deux formations cuticulaires saillantes, ventrale et dorsale (fig. 1 E), il n'y a pas de capsule buccale ; anneau nerveux et pore excréteur situés respectivement à $130 \mu$ et $142 \mu$ de l'apex (fig. $1 \mathrm{~F}$ ). L'œsophage, de structure uniforme, comprend une partie antérieure étroite longue de $165 \mu$ et une partie postérieure renflée longue de $135 \mu$, présentant vers l'intestin une constriction qui isole le bulbe subsphérique (fig. $1 \mathrm{E}$ ) ; l'intestin, large et riche en lipides de réserve, forme un cæcum qui longe le bulbe latéralement. La queue est longue de $108 \mu$, légèrement incurvée sur la face ventrale ; elle s'amenuise régulièrement puis se termine par une courte portion presque cylindrique à extrémité arrondie (fig. $1 \mathrm{G}$ et $\mathrm{H}$ ).

Discussion. - Un tel œsophage ayant à la fois une structure uniforme et une région postérieure nettement plus large que la région antérieure semble être caractéristique de certains Cucullanoidea (sensu Le Van Hoa 1967) comme les Quimperidae (sensu Inglis 1967); en outre, un cæcum intestinal peut s'observer dans cette famille, comme chez Omeia Hsü 1933, parasite de Batracien.

(2) Nous remercions vivement $\mathrm{M}^{\mathrm{me}}$ Petter et $\mathrm{M}$. Chabaud, qui étudient actuellement le cycle de ce Nématode et qui ont bien vouiu mettre à notre disposition leurs documents. 


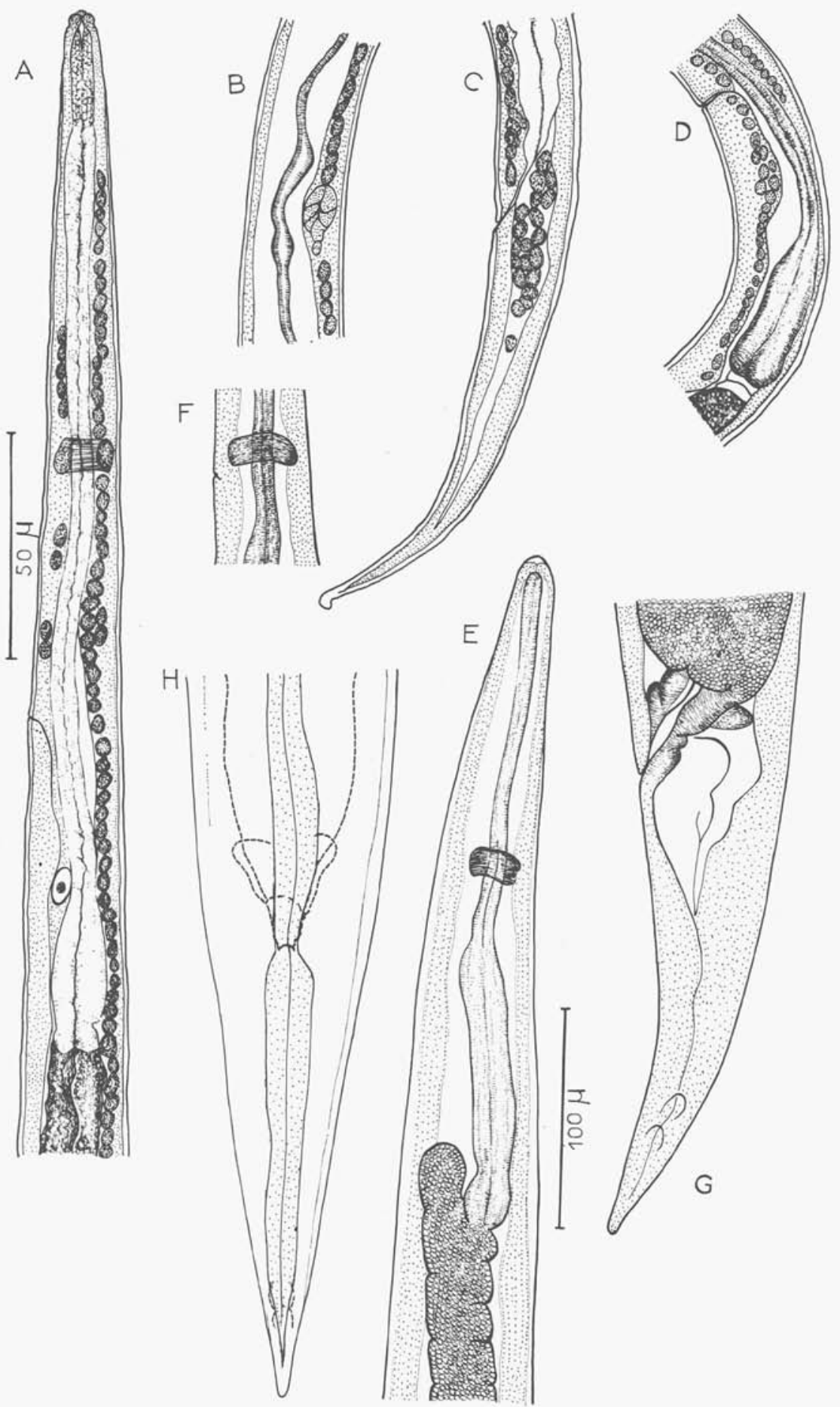

FIG. I. - Larves de Nématodes chez $S$. damnosum; A à D, larve de type Kathlanide: A, région antérieure, vue latérale; B, niveau de l'ébauche génitale; C, région caudale, vue latérale; D, région postérieure de l'œsophage chez un autre spécimen. E à H, larve de type Quimpéride: E, région antérieure, vue médiane; F, pore excréteur, vue latérale ; $\mathrm{G}$, région caudale, vue latérale; $\mathrm{H}$, idem, vue ventrale 


\section{II. - Recherche des adultes.}

Les adultes pouvant correspondre à ces deux types de larves ont été recherchés chez divers Poissons et Batraciens, trouvés aux mêmes endroits que les Simulies parasitées.

\section{A. Poissons.}

Les Poissons disséqués ne renfermaient aucun adulte de Kathlanidae ou de Quimperidae, mais les trois espèces pêchées [Alestes baremose (Joamis 1835), A. nurse (Rüppel 1832), A. leuciscus Günther 1867 (3)] ne représentent qu'une très faible partie de la faune ichtyologique des rivières. Leur vessie natatoire contenait très fréquemment de nombreux exemplaires de la larve de type Quimperide, mais plus grande et avec un cæcum plus développé que chez la Simulie (fig. $2 \mathrm{~A}$ à $\mathrm{E}$ ).

L'abondance de ce matériel conservé en collection a permis de faire une étude de la tête de ce type larvaire en vue apicale. La bouche est triangulaire, dépourvue de lèvres, mais bordée par deux formations cuticulaires saillantes, dorsale et ventrale, présentant chacune une double concavité sur la face interne; les quatre papilles du cycle labial externe sont accolées aux quatre papilles céphaliques et sont situées à la base des formations dorsale et ventrale ; les amphides sont relativement de grande taille (fig. $2 \mathrm{D}$ et $\mathrm{E}$ ).

Mensurations : longueur $2,45 \mathrm{~mm}$, largeur $80 \mu$; œsophage total $380 \mu$; pharynx $52 \mu$, œsophage postérieur renflé $205 \mu$; cæcum $125 \mu$ de long ; anneau nerveux et pore excréteur respectivement à 160 et $170 \mu$ de l'apex; queue longue de $100 \mu$ (4).

\section{B. Batraciens. Description d'un Quimperide, Gendria leberrei, n. sp., parasite de Bufo regularis (fig. 3 ).}

De nombreux Crapauds (Bufo regularis) ont été disséqués ; 10 de Cucullanoidea, représentant une nouvelle espèce, a été récolté dans l'intestin de l'un d'entre eux, à la Leraba. L'absence de cæcum intestinal chez ce spécimen ne permet vraisemblablement pas de le rattacher à la larve de type Quimperide, mais l'existence de cæcum larvaire

(3) Nous remercions vivement M. Daubenton qui a eu la gentillesse de déterminer ces Poissons.

(4) Dans la vessie natatoire des mêmes Poissons, un autre type de larve se trouvait en abondance dans les deux gîtes; comme ses relations avec les types précédents ne peuvent être précisées et que nous ne savons pas à quelle famille elle se rapporte, il semble préférable d'en donner simplement une description (fig. II F à $\mathrm{H}$ ). Le corps de la larve est grêle $(700 \mu$ de long et $7 \mu$ de large), avec deux petites ailes latérales se terminant un peu en avant des extrémités; tête avec deux formations cuticulaires, dorsale et ventrale, saillantes; capsule buccale à peine marquée. Oesophage long de $210 \mu$, comportant une portion antérieure étroite et cylindrique de $120 \mu$, avec différenciation pharyngée de $30 \mu$ de long, et une portion postérieure renflée, simulant un bulbe particulièrement allongé ( $60 \mu$ de long), presque cylindrique et sans valvules ni granules de sécrétion et rappelant l'organite de la larve de type Kathlanide (fig. II F). Anneau nerveux et pore excréteur respectivement à $90 \mu$ et $100 \mu$ de l'apex. Ebauche génitale à $190 \mu$ du début de l'intestin (fig. II G). Queue longue de $80 \mu$ avec segment terminal presque cylindrique, à extrémité arrondie (fig. II H). Noyaux de la couche musculaire bien visibles et volumineux. 


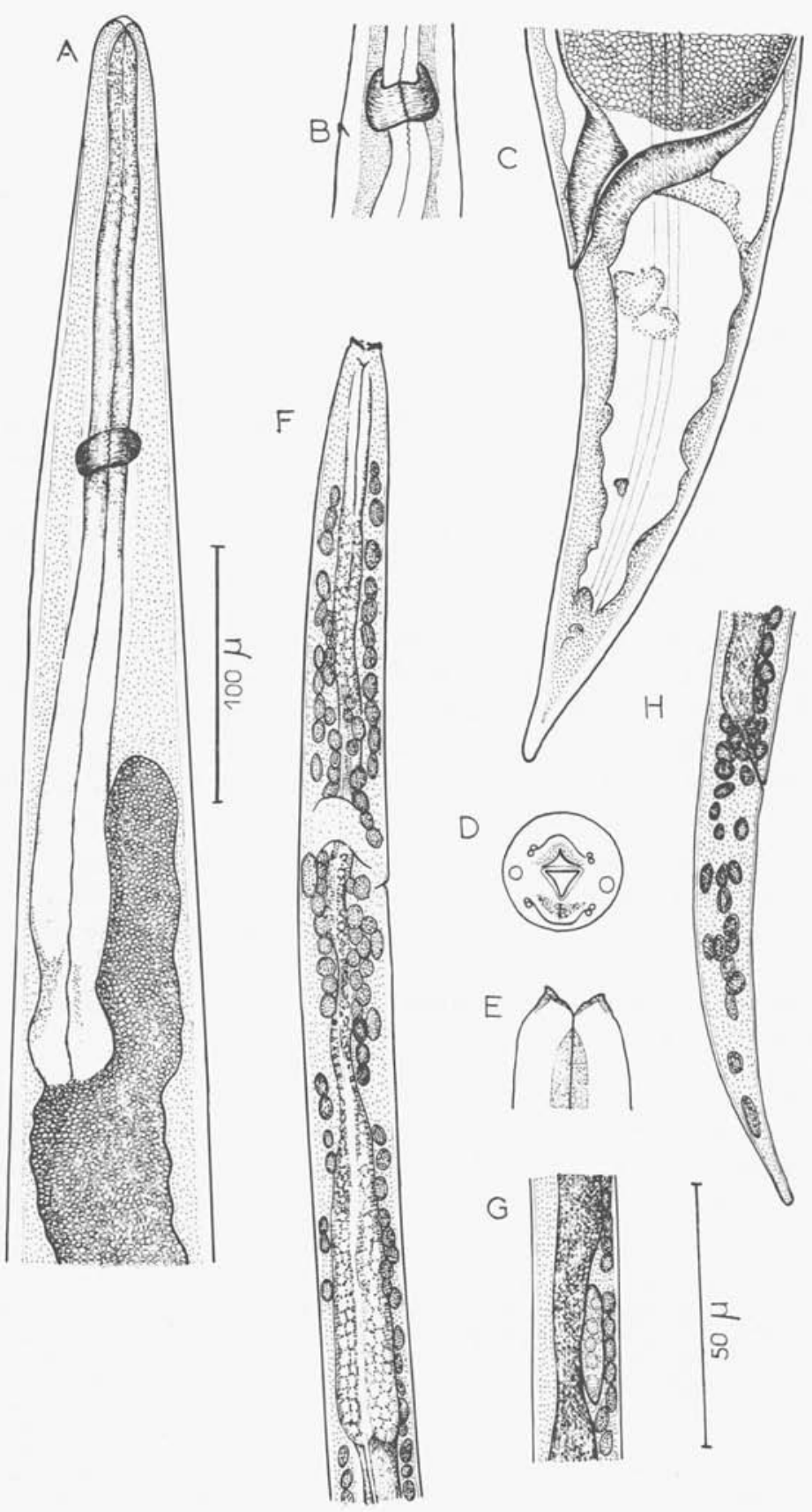

FIG. II. - Larves de la vessie natatoire des Alestes; A à E, larve de type Quimpéride: $\mathrm{A}$, région antérieure, vue médiane; $\mathrm{B}$, pore excréteur, vue latérale ; C, région caudale, vue latérale; D et E, tête, vue apicale et vue latérale. $\mathrm{F}$ à $\mathrm{H}$, larve non identifiée: $\mathrm{F}$, région antérieure, vue latérale ; $\mathrm{G}$, région génitale; $\mathrm{H}$, région caudale, vue latérale 
transitoire chez certains autres Nématodes, par exemple les Ascarides, ne permet pas non p'us d'éliminer totalement cette hypothèse. Il est donc préférable d'en donner la description.

Nématode ténu, long de 9,4 mm et large de $190 \mu$, dépourvu d'ailes latérales, mais possédant une vésicule céphalique assez courte $(110 \mu$ de long et $18 \mu$ d'épaisseur). La tête est caractérisée par la présence d'un revêtement cuticulaire très épais qui arrive directement au contact de l'œsophage ; cet épaississement est creusé en son milieu pour former une sorte de cavité buccale; la bouche est entourée par six replis pétaloïdes non charnus ; le fond de la cavité buccale a une section triangulaire, à parois convexes, et est fermée par trois dents pharyngiennes inégales : la dent dorsale est plus grosse et plus postérieure que les deux dents latéro-ventrales; six papilles labiales internes de petite taille à gros filet nerveux ; papilles labiales externes et céphaliques complètement fusionnées deux à deux : leurs quatre gros filets nerveux repoussent la cuticule péribuccale et forment quatre dômes ; les amphides ne dépassent pas le niveau du plateau cuticulaire (fig. $3 \mathrm{D}, \mathrm{E}, \mathrm{F})$. Anneau nerveux à $190 \mu$ de l'apex ; pore excréteur et deirides très postérieurs, respectivement à 510 et $455 \mu$ de l'apex ; les deirides sont développées, en forme de crochet (fig. 3 B et C). Esophage claviforme, assez court $(565 \mu)$, avec différenciation pharyngée ; au niveau de l'anneau nerveux, la structure de l'œsophage se modifie légèrement: il reste musculaire mais présente aussi de nombreuses granulations de sécrétion (fig. $3 \mathrm{~A}$ ).

La queue est assez courte $(180 \mu)$, large mais se rétrécit brusquement à son extrémité ; ventouse dépourvue d'anneau chitinoïde située à $650 \mu$ de la pointe caudale ; spicules égaux, longs de $340 \mu$, fortement sclérifiés; la pointe de chaque spicule a un diamètre brusquement plus étroit que celui du corps du spicule et se termine par une lame cuticulaire conique (fig. $3 \mathrm{G}$ et $\mathrm{H})$; gubernaculum petit $(50 \mu$ de long) mais bien sclérifié.

Les papilles sont petites sauf la papille impaire précloacale située à $75 \mu$ du cloaque ; cinq paires de papilles précloacales s'étageant du cloaque à la ventouse ; cinq paires de papilles postcloacales, les deux moyennes ayant une position ventro-latérale (fig. $3 \mathrm{G}$ ).

Discussion. - La vésicule céphalique, l'œsophage relativement court et claviforme, la ventouse précloacale non chitinisée constituent un ensemble de caractères qui placent notre spécimen dans la sous-famille des Gendriinae Chabaud, Campana-Rouget et Brygoo, 1960, telle qu'elle est définie par Inglis (1967).

La présence très caractéristique de trois dents pharyngiennes avec dent dorsale plus développée rapproche notre matériel de l'espèce Gendria tilapiae Baylis 1930 (cf. Chabaud 1956) ; toutefois, il s'en distingue facilement par la présence de six lèvres, d'un épaississement cuticulaire péribuccal ayant l'aspect d'un diadème grâce aux quatre papilles externes saillantes.

Nous proposons pour cette nouvelle espèce le nom de Gendria leberrei n. sp., en hommage au Docteur Le Berre. 

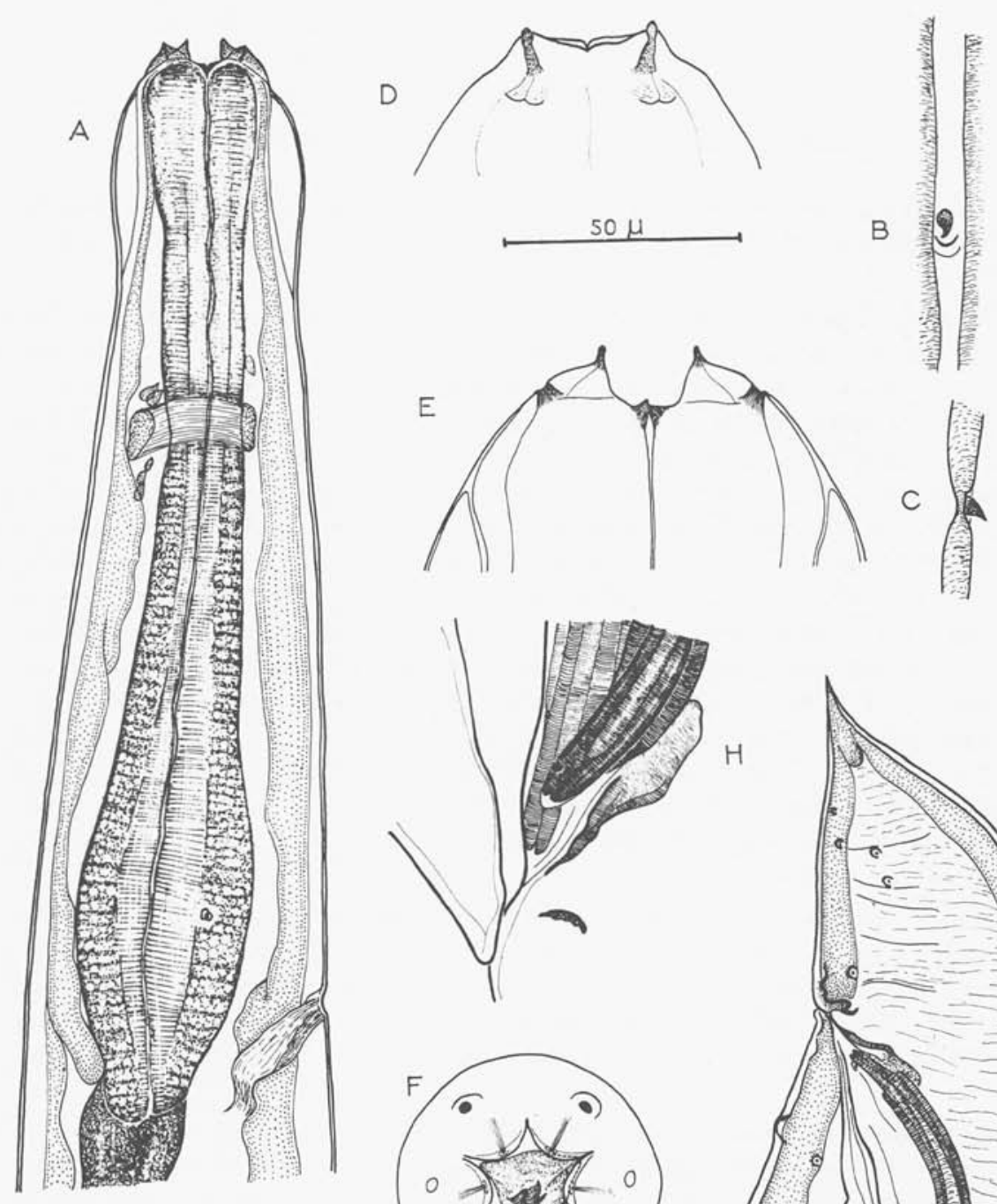


\section{Conclusion.}

Le fait que des larves soient récoltées chez $S$. damnosum n'indique pas obligatoirement que cet Insecte soit un hôte intermédiaire; il peut jouer le rôle d'un hôte d'attente. En effet, un tel cycle à hôte d'attente a déjà été observé chez un Kathlanide, Megalobatrachonema campanae, l'hôte d'attente étant cette fois un Oligochète (Chabaud, in Grassé 1965).

Par contre, aucun cycle de Quimperidae n'est connu; mais il est probable que cette famille primitive, parasite de Poissons, ait une biologie plus proche de celle des Kathlanides que de celle des Seuratidae, famille spécialisée parasite de Chiroptères, dans laquelle l'Insecte est un véritable hôte intermédiaire (Le Van Hoa 1966).

Il est difficile de savoir si ces hôtes d'attente sont, comme le notait Chabaud en 1962, physiologiquement ou écologiquement obligatoires, servant uniquement dans ce dernier cas à augmenter la concentration de larves naturellement très dispersées dans le milieu aquatique.

La même question peut se poser au sujet du rôle des Poissons qui renferment des larves de Quimperide ; il n'est pas possible actuellement de savoir s'ils constituent une phase intermédiaire obligatoire du cycle.

\section{Bibliographie}

Balay (G.), 1968. - Bio-écologie des $q$ de Similium damnosum Theobald, 1903, dans les foyers d'Onchocercose de la Volta Blanche, de la Volta Rouge et de la Sissili. Rapport O.C.C.G.E. Centre Muraz (à paraître).

Baylis (H. A.), 1930. - Mission Saharienne Augiéras-Draper, 1927-1928. Parasitic Nematodes. Bull. Mus., 2, 117-130.

Chsbaud (A.-G.), 1956. - Structure céphalique de Gendria tilapiae Baylis, 1930. Ann. Parasit. hum. comp., 31, 310-311.

Chabaud (A.-G.), in Grassé (P.-P.), 1965. - Traité de Zoologie. Nemathelminthes, 4 (3), 1.497 pp.

Chabaud (A.-G.), Brygoo (E. R.), Petter (A. J.), 1962. - Cycle évolutif de l'Ascaride des Caméléons malgaches. Bull. Soc. Zoo. France, 87, 515-532.

Chabaud (A.-G.), Campana-Rouget (Y.), Brygoo (E. R.), 1960. - Les Nematodes Seuratoidea. Ann. Parasit. hum. comp., 35, 316.

Chabaud (A.-G.) et Golvan (Y. J.), 1957. - Megalobatrachonema campanae n. sp. (Nematoda Kathlaniinae), parasite de Tritons de la région parisienne. Ann. Parasit. hum. comp., 32, 243-263.

Hsu (H. F), 1933. - On some parasitic Nematodes collected in China. Parasitology, 24, 512-541.

INGLIS (W. G.), 1967. - The relationship of the Nematoda superfamily Seuratoidea. J. Helm., 41, 115-136. 
LE VAN HoA, 1966. - Cycle évolutif de Seuratum nguyenvanaii Le Van Hoa, 1964, parasite de la Musaraigne, Suncus murinus (L.) au Viêt-Nam. Bull. Soc. Path. exot., 59, 124-128.

LE VAN HoA, 1967. - Relation entre les Seuratidae et les Cucullanidae par l'intermédiaire d'un nouveau Nematode Campanarougetia, campanarougetae, n.g., n. sp., parasite des Anguilles du Sud Viêt-Nam. Bull. Soc. Path. exot., 60, 393-398.

LEWIS (D. J.), 1953. - Simulium damnosum and its relation to onchocerciasis in the Anglo-egyptian Sudan. Bull. ent. Res., 43, 597-644.

Lewis (D. T.) et MarR (J. D. M.), 1963. - A Nematode from the malpighian tubes of Simulium damnosum. Trans. R. Soc. trop. Med. Hyg., 57, 238.

MARR (J. D. M.) et LewIS (D. J.), 1964. - Observations on the dry-season survival of Simulium damnosum Theo. on Ghana. Bull. ent. Res., 55, 547-564.

Ovazza (M.), Coz (J.) et Ovazza (L.), 1965. - Etude des populations de Simulium damnosum Theobald, 1903 (Diptera; Simuliidae) en zones et gîtes non permanents. 1. Observations sur les variations de quelques-uns des caractères utilisés dans l'estimation de l'âge physiologique. Bull. Soc. Path. exot., 58, 938-950. 